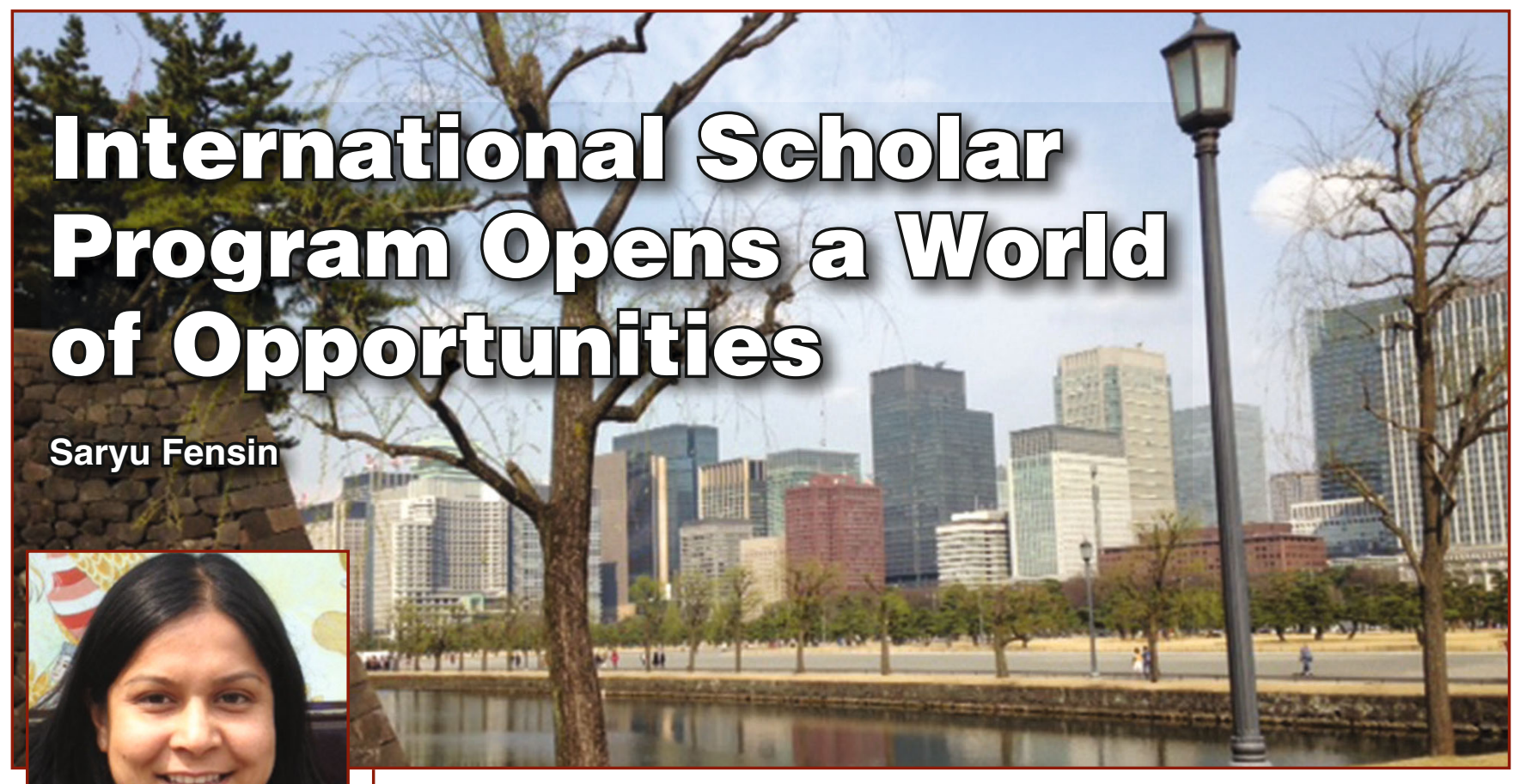

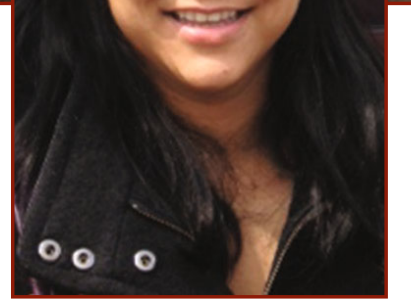

Saryu Fensin
I was honored and grateful to be selected as the 2016 Japan Institute of Metals (JIM)/TMS Young Leaders International Scholar. This award, which is generously supported by the TMS Foundation, enabled me to attend the JIM 2016 Annual Spring Meeting in Tokyo and to visit the National Institute for

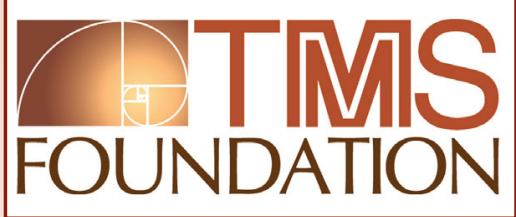

\section{Make a Global} Impact: Support the 2016 TMS Foundation Annual Appeal

Giving promising young minerals, metals, and materials scientists and engineers an opportunity to develop scientific collaborations across cultures is the goal of the TMS Young Leaders International Scholar Program, made possible by the TMS Foundation. Since 2005 both TMS and the Japan Institute of Metals and Materials (JIM) have selected young professionals, through a competitive review of their accomplishments, to travel to the other organization's annual meetings to present scientific papers and participate in learning and networking activities. A similar effort was launched in 2013 with the Federation of European Materials Societies (FEMS).

You can ensure that the TMS Young Leaders International Scholar Program continues to engage future generations of professionals in important global collaborations by donating to the TMS Foundation. You may have already received information about the TMS Foundation's 2016 Annual Appeal that details how the foundation has benefitted scores of young and emerging professionals through its programs. Read it and act today to keep up this good work. Visit the TMS Foundation website at www.TMSFoundation.org to make an online donation or contact Mary Samsa, TMS Foundation \& Public Affairs Manager at msamsa@tms.org for additional information on giving opportunities.

Materials Science (NIMS) in Tsukuba. This experience offered me unique opportunities to not only present my current work, but also to network with colleagues and learn more about Japanese culture.

I am particularly appreciative of the hospitality shown to me by Yoshimasa Kajiwara (JIM) and Yoko Yamabe-Mitarai (NIMS). Kajiwara contacted me months before my trip with an itinerary and detailed maps with directions to my hotel. I was initially apprehensive about making my way from the airport to the hotel and various conference locations using the public transit system, since the signage is primarily in Japanese, but my worries were put to rest by the helpfulness of the people I encountered on this trip.

My first day in Japan included enjoying the wonderful Japanese cuisine and preparing for my visit to NIMS. The following day, my NIMS host, YamabeMitarai, was kind enough to pick me up at the train station in Tsukuba and, after arriving at NIMS, I presented my work on dynamic behavior of materials. This resulted in interesting discussions throughout the day. Yamabe-Mitarai then gave me a tour of the impressive facilities at NIMS and introduced me to other scientists. I was impressed by the abundance of mechanical testing equipment used to measure the response 


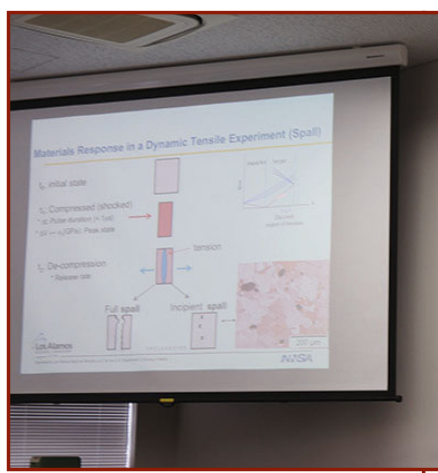

Saryu Fensin delivers her award lecture, "Predicting Dynamic Fracture in Metals and Alloys," at the JIM 2016 Annual Spring Meeting.

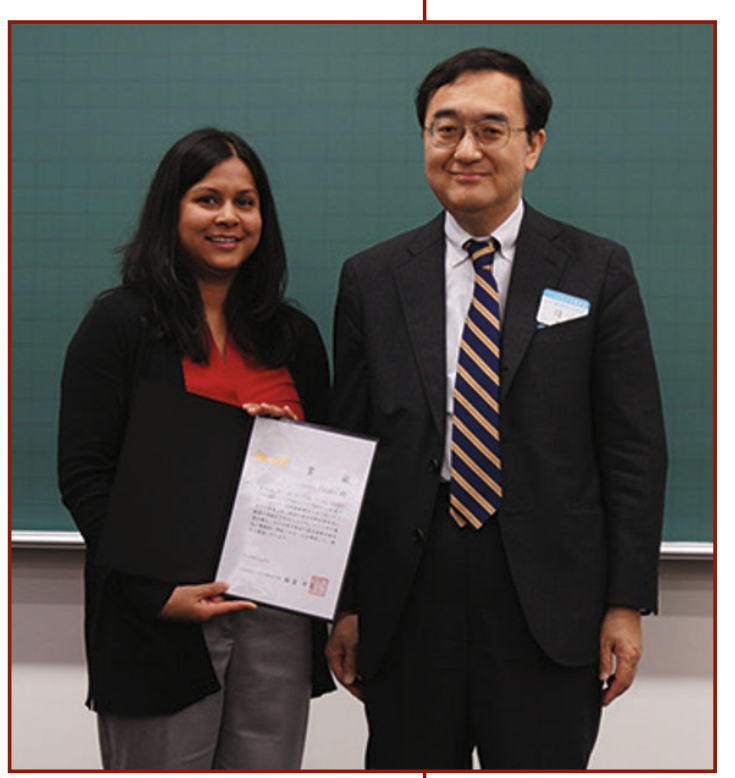

of materials under conditions ranging from fatigue tests to more prevalent quasi-static conditions. During my tour, I was also introduced to some unique sample designs used to study the effect of hydrogen embrittlement on the mechanical properties of materials. Through my discussions with various scientists, I learned about similarities and differences in the challenges that young scientists face in the United States compared with Japan. The day ended with a wonderful Kaiseki

Saryu Fensin receives recognition of her JIM/TMS Young Leaders International Scholar Award from Manabu Enoki, Professor, University of Tokyo. meal hosted by Yamabe-Mitarai in a traditional Japanese restaurant.

A highlight of the following day was attending the JIM Annual Spring Meeting awards ceremony where I was introduced to the JIM executive board members and treated like a special guest. I also took advantage of an opportunity to participate in a walking tour of Tokyo. What stood out most to me about this wonderful city is the truly unique juxtaposition of traditional and the modern architecture. I ended the day by attending the meeting banquet hosted by JIM, where I met other award recipients and collaborators. As a symposium organizer for the 9th Pacific Rim International Conference on Advanced Materials and Processing (PRICM9), I also had the opportunity to meet co-organizers with whom I had only communicated through email. (PRICM9, hosted by JIM, was held August 1-5, in Kyoto, Japan.)

I concluded my trip by presenting my talk, "Predicting Dynamic Fracture in Metals and Alloys" at a special session held the following day.

Yamabe-Mitarai, my host who warmly welcomed me to Japan, was an amazing resource throughout my experience. This unforgettable trip would also not have been possible without the great relationship between JIM and TMS and the support of the TMS Foundation. I thank everyone who was involved in giving me this chance to meet new colleagues, have interesting discussions, and explore Japan.

\section{Saryu Fensin is a staff member at Los Alamos National Laboratory and past chair of the TMS Young Professional Committee. She is also the recipient of the 2014 Functional Materials Division Young Leaders Professional Development Award.}

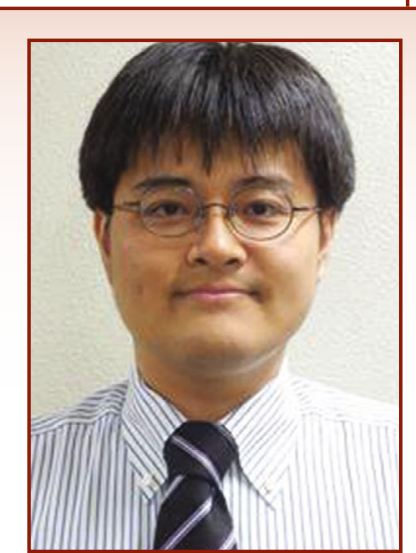

Motomichi Koyama

\section{Meet the 2016 JIM Representative}

Motomichi Koyama was selected by JIM as its representative in the 2016 Young Leaders International Scholar Program. JIM supported his travel to the TMS 2016 Annual Meeting \& Exhibition, February 14-18, in Nashville, Tennessee, where he presented his lecture, "Effective Utilization of Martensite in Fe-High Mn Austenitic Steels: Aspects of Deformation-Induced Reverse Transformation." Koyama has been an assistant professor at Kyushu University since 2013. From 2012 to 2013, he was a senior researcher at Max Planck Institute fur Eisenforschung. He received his Ph.D. from Tsukuba University in March 2012. His research is on effects of martensitic transformation, deformation twinning, and strain aging on fatigue crack growth, hydrogen embrittlement, and work hardening.
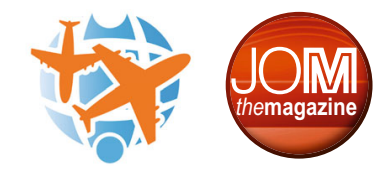